\title{
Research and application of intelligent control method of small type loader actuator
}

\author{
Youping Gong ${ }^{1,2,3, a, *}$, Ke Jiang ${ }^{2, b}$, Tao Jin ${ }^{1, c}$, Huipeng Chen ${ }^{2, d}$, Dan Li $^{3, e}$ \\ ${ }^{1}$ Department of Chemical Engineering, Zhejiang University, Hangzhou, china \\ ${ }^{2}$ Department of Mechanical, Hangzhou Dianzi University, Hangzhou, china \\ ${ }^{3}$ Zhejiang Kaiji Auto Parts Manufacturing Co. Ltd. postdoctoral workstation,Yiwu,Zhejiang,china \\ a gypcad@163.com, b jiangke@hdu.edu.cn, c cejintao@zju.edu.cn, ${ }^{\mathrm{d} l y n x}$ lchen@163.com, ${ }^{\mathrm{C}}$ lidan@163.com \\ *Youping Gong
}

Keywords: D-H method; Fuzzy adaptive PID Controller; Model Simulation

\begin{abstract}
This paper studies the control system of self-excavated excavator, which is based on the working device of the small type loader. Firstly, the kinematics model of the working device is established by D-H method, so that the trajectory planning of the vehicle speed, boom oil cylinder and bucket oil cylinder drive equation is obtained. Combined with the advantages of PID control and fuzzy control, the fuzzy adaptive PID controller of the actuator is designed. Finally, the simulation model of the hydraulic system position controller is built in Simulink. The simulation results show that the displacement error of the actuator is within $0.5 \mathrm{~mm}$.
\end{abstract}

\section{Introduction}

Operating conditions of loader actuator are mainly excavation materials, heavy transport and discharge and so on. Among them, the excavation of materials is the process of the required largest power of the process. The excavation operation of the non-intelligent loader is accomplished by manipulating the boom handle or the bucket handle by the operator, not only the work intensity is large, but also the operation efficiency is low[1][2]. The current operating methods used by the loader include single shovel method and matching shovel method, the single shovel method let the bucket blade insert the bottom of object, when the depth equal the length of bucket bottom, the loader stops moving forward, and then the turning cylinder moving to upward and realize bucket flipping. Matching shovel method is that when loader body moving forward, the boom is enhancing bucket is turning around at the same time.Although the single shovel method is simple to operate, the driver's operating level is not demanding, but its operating resistance is 2 to 3 times more than the matching shovel method [3], which means that the more energy is consumed. The ideal shovel operation should not only meet the lower energy consumption rate, but also pay attention to reasonable labor costs. Therefore, if you can use the robot technology and intelligent control technology to achieve the automatic excavator digging operations to eliminate the human and energy waste caused by differences level of driver's operation , improve the efficiency of the loader, it is undoubtedly on the modernization and social development is of great significance and value.

\section{The dynamic digging model construction of loader}

The working device of the loader is composed of bucket, boom, rocker, connecting rod, bucket oil cylinder and boom oil cylinder, as shown in Fig 1.1.The entire working device is connected to the front frame and the bucket is articulated to the bucket oil cylinder through the connecting rod and rocker for loading and unloading the material; The boom is articulated to the front frame and the boom oil cylinder to lift the bucket. In the case of excavation, the loader can be regarded as having three degrees of freedom, which are the translation of the body, the lifting of the boom and the turning of the bucket. 


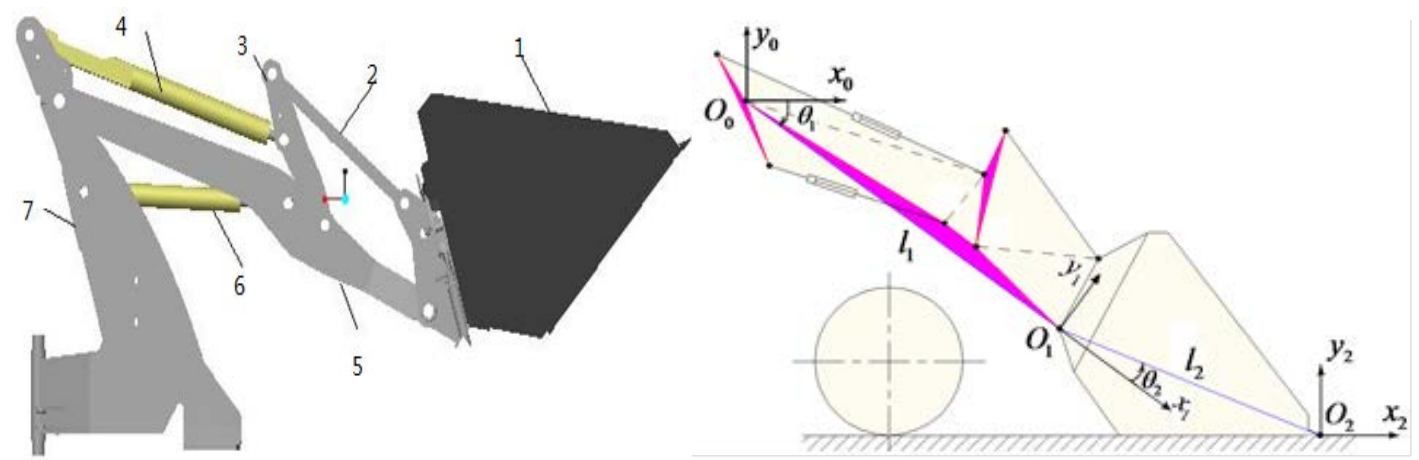

1.Bucket; 2.Connecting rod; 3. rocker;

Fig 1.2 Loader working device D-H coordinate system

4.bucket oil cylinder; 5. power arm;

6. boom lift oil cylinder; 7. Front frame

Fig1.1 Loader working device

The working device of the loader can be viewed as a 2-DOF robot operating arm whose position of the end is controlled by the change in the length of the boom oil cylinder and the bucket oil cylinder. Therefore, the robot kinematics method can be used to analyze the loader working device [4]. The D-H method is used to model the loader working device. The following steps $i(i=0,1,2)$ can be used to establish a coordinate system $O_{i} X_{i} Y_{i} Z_{i}$ that is fixed to the bars.

(1) $O_{0} 、 O_{1} 、 O_{2}$ is the origin of the three coordinate system respectively, the rod $O_{0} O_{1}$ represents the boom, the rod $O_{1} O_{2}$ represents the bucket;

(2) Take $Z_{0}$-axis $、 Z_{1}$-axis $、 Z_{2}$-axis 3 axis parallel, and the points are perpendicular to the plane $O_{0} X_{0} Y_{0}$;

(3) $X_{0}$-axis has been determined; $X_{1}$-axis and $O_{0} O_{1}$ are same line, and along $O_{0} O_{1}$ direction; $X_{2}$-axis and $O_{1} O_{2}$ are same line, and along $O_{1} O_{2}$ direction;

(4) According to the principle of $Y_{i}=Z_{i} \times X_{i}$, Respectively, to determine $Y_{0} 、 Y_{1} 、 Y_{2}$ axis.

According to the above steps, the $\mathrm{D}-\mathrm{H}$ coordinate system of the loader working device is established as shown in Figure1.2.

After establishing the D-H coordinate system, it is necessary to determine the four geometric parameters $\theta_{i} 、 \alpha_{i} 、 d_{i} 、 a_{i}$ of the bar to realize the transformation between the coordinate system. These parameters establish a $4 \times 4$ homogeneous transformation matrix for the rod coordinate system at each joint, indicating its relation to the previous rod coordinate system. The value of the D-H parameter of the loader working device is shown in Table 1.1.

Table1.1 loader working device D-H parameter table

\begin{tabular}{ccccc}
\hline joint $i$ & $\theta_{i}$ & $\alpha_{i}$ & $d_{i}$ & $a_{i}$ \\
\hline 1 & $\theta_{1}$ & 0 & 0 & $l_{1}$ \\
2 & $\theta_{2}$ & 0 & 0 & $l_{2}$ \\
\hline
\end{tabular}

The meaning of the parameters in the table is:

$\theta_{i}$-Around the $Z_{i-1}$ axis, the angle turning from $X_{i-1}$-axis to $X_{i}$-axis according to the right hand rules;

$\alpha_{i}$-Around the $X_{i}$ axis, the angle turning from $Z_{i-1}$-axis to $Z_{i}$-axis according to the right hand rules;

$d_{i}$-The distance from the $Z_{i-1}$-axis to $Z_{i}$-axis, along the $X_{i}$-axis is positive;

$a_{i}$-the distance from the $X_{i-1}$-axis to $X_{i}$-axis, along the $Z_{i-1}$-axis is positive;

Substituting the D-H parameters in Table 1.1 into D-H equation, then the transformation matrix of the boom relative to the base and the bucket relative to the boom can be described as equation (1.1) and (1.2) respectively: 


$$
\begin{aligned}
{ }^{0} A_{1} & =\left[\begin{array}{cccc}
\cos \theta_{1} & -\sin \theta_{1} & 0 & l_{1} \cos \theta_{1} \\
\sin \theta_{1} & \cos \theta_{1} & 0 & l_{1} \sin \theta_{1} \\
0 & 0 & 1 & 0 \\
0 & 0 & 0 & 1
\end{array}\right] \\
{ }^{1} A_{2} & =\left[\begin{array}{cccc}
\cos \theta_{2} & -\sin \theta_{2} & 0 & l_{2} \cos \theta_{2} \\
\sin \theta_{2} & \cos \theta_{2} & 0 & l_{2} \sin \theta_{2} \\
0 & 0 & 1 & 0 \\
0 & 0 & 0 & 1
\end{array}\right]
\end{aligned}
$$

Then the homogeneous transformation matrix of the bucket end relative to the base coordinate system can be presented as (1.3) as following:

$$
{ }^{0} T_{2}={ }^{0} A_{1}{ }_{1}^{1} A_{2}=\left[\begin{array}{cccc}
\cos \left(\theta_{1}+\theta_{2}\right) & -\sin \left(\theta_{1}+\theta_{2}\right) & 0 & l_{2} \cos \left(\theta_{1}+\theta_{2}\right)+l_{1} \cos \theta_{1} \\
\sin \left(\theta_{1}+\theta_{2}\right) & \cos \left(\theta_{1}+\theta_{2}\right) & 0 & l_{2} \sin \left(\theta_{1}+\theta_{2}\right)+l_{1} \sin \theta_{1} \\
0 & 0 & 1 & 0 \\
0 & 0 & 0 & 1
\end{array}\right]
$$

According to Eq. (1.3), the positive solution of the bucket tooth tip position can be easily obtained, and the inverse solution of the tooth tip position is obtained by using this positive solution, that is the joint angle $\theta_{1}, \theta_{2}$ in Table1.1.

Setting coordinates of the loader bucket tip $P$ is $\left(P_{x}, P_{y}, 0\right)$, from the formula (1.3) kinematics positive solution can be obtained:

$$
\left\{\begin{array}{l}
P_{x}=l_{2} \cos \left(\theta_{1}+\theta_{2}\right)+l_{1} \cos \theta_{1} \\
P_{y}=l_{2} \sin \left(\theta_{1}+\theta_{2}\right)+l_{1} \sin \theta_{1} \\
P_{z}=0
\end{array}\right.
$$

Inverse kinematics can be obtained by reverse solution from the above equation(1.5):

$$
\left\{\begin{array}{l}
\theta_{1}=\alpha \pm \cos ^{-1} \frac{P_{x}^{2}+P_{y}^{2}+l_{1}^{2}-l_{2}^{2}}{2 l_{1} \sqrt{P_{x}^{2}+P_{y}^{2}}} \\
\theta_{2}=\sin ^{-1} \frac{\sqrt{P_{x}^{2}+P_{y}^{2}} \sin \left(\alpha-\theta_{1}\right)}{l_{2}} \\
\alpha=\tan ^{-1} \frac{P_{y}}{P_{x}}
\end{array}\right.
$$

According to the combined excavation method, the space position of the bucket tooth tip depends on the movement of the working device itself and its horizontal movement with the vehicle body during the excavation operation. The predetermined excavation trajectory consists of a series of points $(X, Y)$, then $X=P_{x}+s, Y=P_{y}, s$ is the distance of the loader traveling in the direction of $X$, then the excavation trajectory can be obtained( 1.6):

$$
\left\{\begin{array}{l}
\theta_{1}=\alpha-\cos ^{-1} \frac{(X-s)^{2}+Y^{2}+l_{1}^{2}-l_{2}^{2}}{2 l_{1} \sqrt{(X-s)^{2}+Y^{2}}} \\
\theta_{2}=\sin ^{-1} \frac{\sqrt{(X-s)^{2}+Y^{2}} \sin \left(\alpha-\theta_{1}\right)}{l_{2}} \\
\alpha=\tan ^{-1} \frac{Y}{X-s}
\end{array}\right.
$$




\section{1Control system model of loader actuator}

The core of the actuator control system is the Electra-hydraulic proportional closed-loop position control system, which consists of a hydraulic source, a control mechanism, an actuator and a controller. The displacement sensor detects the displacement of the hydraulic cylinder and feeds back to the input of the proportional amplifier. Compared with the given electrical signal, the obtained deviation signal is amplified to control the throttle opening of the proportional directional valve until the deviation is zero. The output displacement of the hydraulic cylinder corresponds to the input electrical signal and is proportional to the input electrical signal.

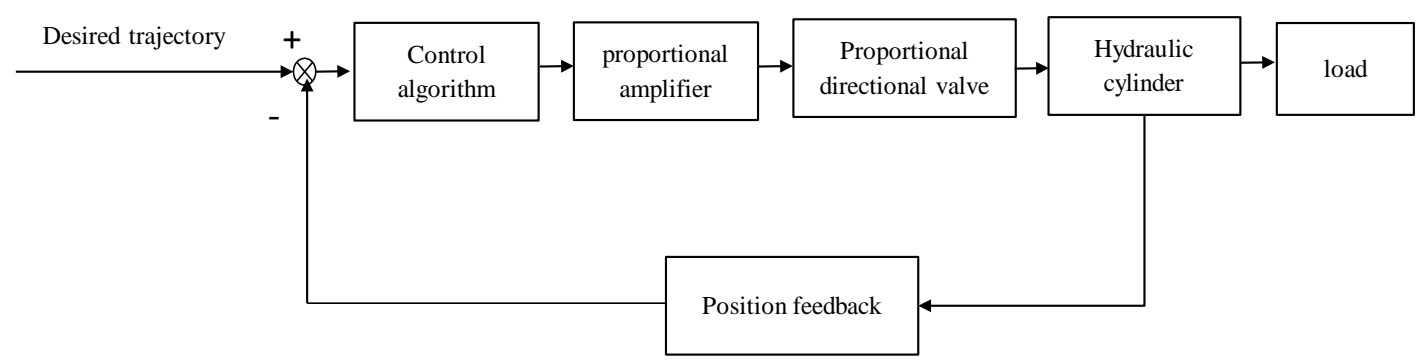

Figure 2.1 Block diagram of electro-hydraulic proportional position control system The block diagram of the position control system is shown in Figure2.2

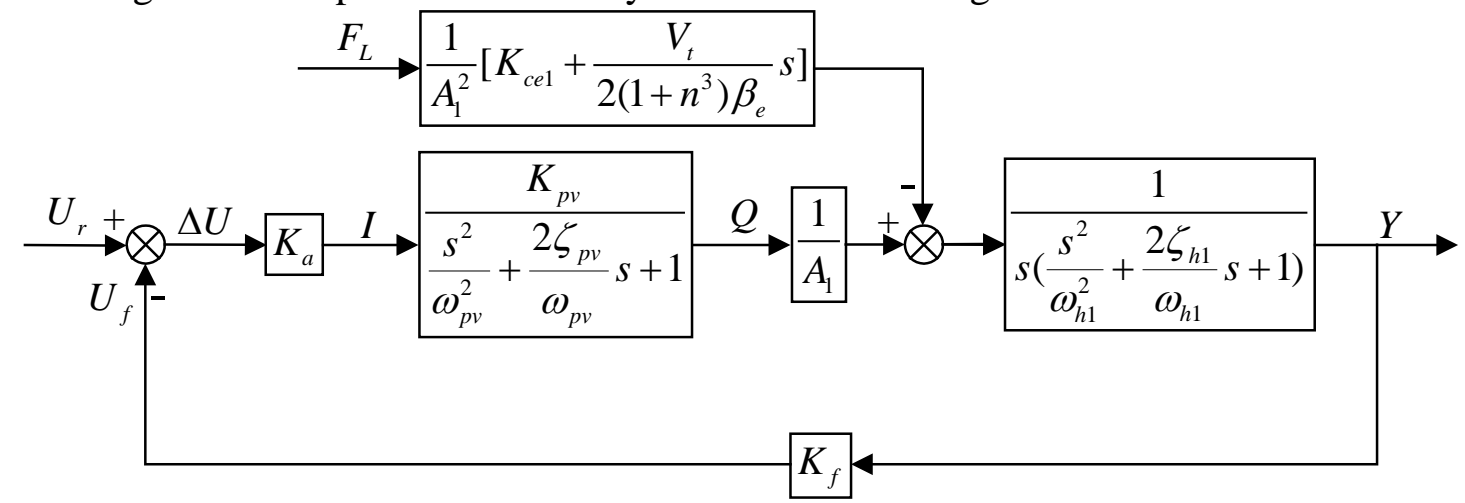

Figure 2.2 Block diagram of the position control system when $\dot{y}>0\left(x_{v}>0\right)$

Open - loop Transfer Function of Piston Displacement of Hydraulic Cylinder to Input Voltage Signal can be obtained from figure2-2:

$$
G_{K}(s)=\frac{K_{a} K_{p v} K_{f}}{A_{1} s\left(\frac{s^{2}}{\omega_{p v}^{2}}+\frac{2 \zeta_{p v}}{\omega_{p v}} s+1\right)\left(\frac{s^{2}}{\omega_{h 1}^{2}}+\frac{2 \zeta_{h 1}}{\omega_{h 1}} s+1\right)}
$$

Under normal circumstances, when the natural frequency of the proportional valve is much higher than the natural frequency of the power mechanism, the proportional valve can often be reduced to proportional links, Thus, the open-loop transfer function can be approximated as:

$$
G_{K}(s)=\frac{K_{a} K_{p v} K_{f}}{A_{1} s\left(\frac{s^{2}}{\omega_{h 1}^{2}}+\frac{2 \zeta_{h 1}}{\omega_{h 1}} s+1\right)}
$$

The closed-loop transfer function of the system:

$$
G_{B}(s)=\frac{Y}{U_{r}}=\frac{K_{a} K_{p v}}{A_{1} s\left(\frac{s^{2}}{\omega_{h 1}^{2}}+\frac{2 \zeta_{h 1}}{\omega_{h 1}} s+1\right)+K_{a} K_{p v} K_{f}}
$$




\subsection{The actuator fuzzy adaptive PID control simulation model}

The basic idea of fuzzy adaptive PID control is to combine PID control and fuzzy control. Based on the knowledge representation of fuzzy mathematics and fuzzy language ${ }^{[5]}$, the condition and operation of fuzzy rules are expressed, and the fuzzy control rules and related information are stored Into the computer knowledge base, according to the system response, the use of fuzzy reasoning automatically realize the best adjustment of the three parameters of PID.The Structure of Fuzzy Adaptive PID Controller for Loader Working Device is shown in Figure 2.3.
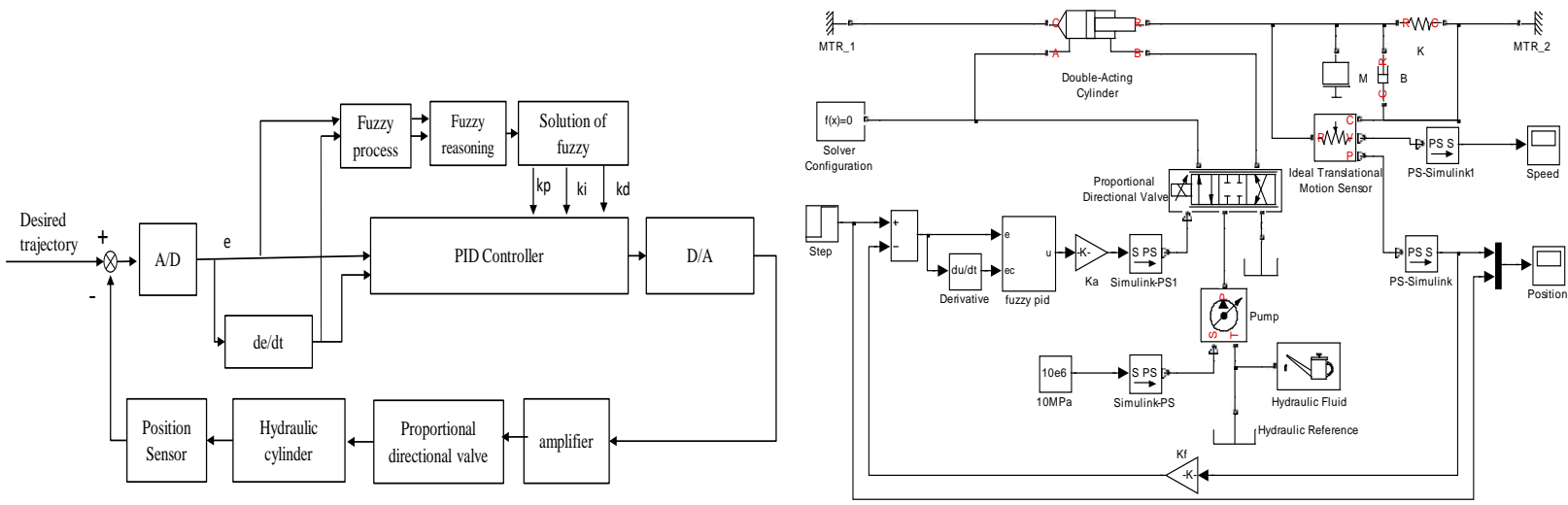

Figure 2.3 Fuzzy adaptive PID controller structure Figure2. 4 Fuzzy adaptive PID hydraulic position

control system simulation block diagram

The fuzzy adaptive PID controller is applied to the SimHydraulics hydraulic model of the loader working device, and the rationality of the controller design is verified. The simulation model is shown in Figure2.4.

After repeated testing, the initial PID parameter is set to $K_{P 0}=11, K_{I 0}=150, K_{D 0}=0.01$. The step signal test simulation is carried out on the fuzzy adaptive PID control system. The response result is shown in Figure 2.5.

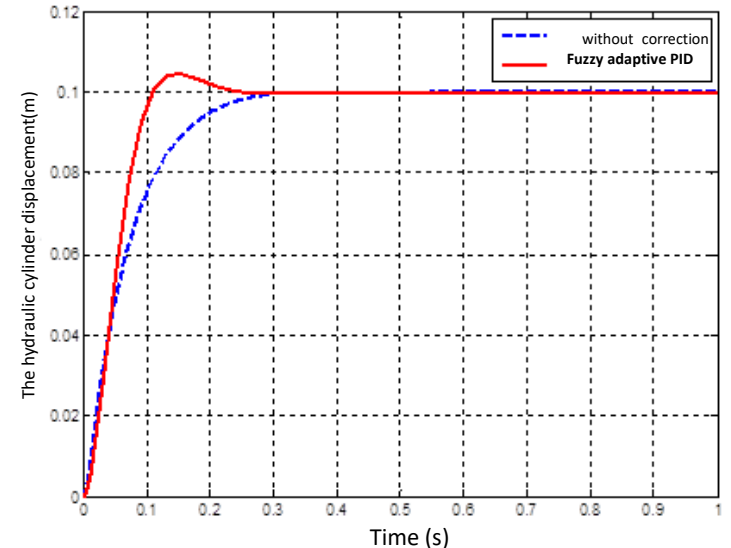

Figure 2.5 Fuzzy adaptive PID control step

response curve

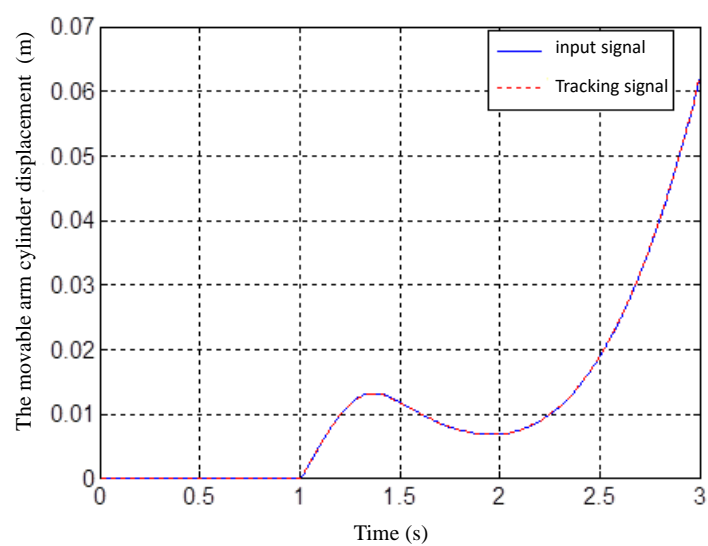

Figure 2.6 The displacement tracking simulation

under the fuzzy adaptive PID controller

The results of step response simulation are shown that with fuzzy adaptive PID correction, the system response speed is high, the accuracy is high, the overshoot is small, the system is stable and the control effect is better. According to the results of the second chapter trajectory planning, the position control system simulation of the actual signal of the boom oil cylinder and the bucket oil cylinder is carried out, and the tracking effect of the fuzzy adaptive PID controller is observed to lay the foundation for the autonomous excavation of the loader. The displacement tracking simulation and error analysis of boom oil cylinder and bucket oil cylinder are shown in Figure 2.6 and2.7 respectively. From the simulation results can be seen, the result of the loader working device hydraulic system fuzzy adaptive PID position control is quite satisfactory. It can be clearly seen from the error diagram that the maximum error of the trajectory tracking of the boom oil cylinder is 
$4.2 \times 10-4 \mathrm{~m}$ and the maximum error of the track of the bucket cylinder is $1.7 \times 10-4 \mathrm{~m}$, both of which occur in the hydraulic cylinder time. This is because the hydraulic pump suddenly opened, the oil pressure of the system rises instantaneously causing the shock wave through the proportional valve reached the hydraulic cylinder, and it will inevitably produce a certain impact. Although there will be some track tracking error for two hydraulic cylinders in the start of a period of time ,the accuracy is controlled within $0.5 \mathrm{~mm}$, indicating that the design of the location control system is reasonable.

\section{Conclusion}

In this paper, the dynamic model of the working device is established and the control system model of the whole working device is established. Based on the advantages of PID control and fuzzy control, a fuzzy adaptive PID controller is designed for the position control system of the working device. The simulation results show that the boom cylinder and the bucket cylinder can move according to the given trajectory, and the displacement error is controlled within a reasonable range, which lays the foundation for the next step of the independent excavator simulation.

\section{Acknowledgement}

The paper was support by the National Natural Science Foundation of China (61100101, 51475129, 51275141, and 51305113), the paper was also supported by Key Discipline of The Ocean Techtronic Equipment's Technology. The paper was supported by The Southern Taihu elite project: The R \& D and manufacture of high performance nuclear power using ring chain and Zhejiang Province postdoctoral excellent project :The design and application of industrial robot and the establishment of an industrial robot design and simulation platform.

\section{References}

[1] Liu Jianhua, Gong Youping, Chen Guojin, Chen Huipeng. Modeling and simulation of loader working device based on SimMechanics. Proceedings 2011 International Conference on Transportation, Mechanical, and Electrical Engineering, TMEE 2011, 2011: 2054-2057.

[2] Chen Huipeng, Liu Jianhua, Chen Guojin, Gong Youping. Application of Single Neuron PID in the control of loader working device. Applied Mechanics and Materials, 2012, 130-134: 715-718.

[3] Ahmad Hemami. Motion trajectory study in the scooping operation of an LHD-loader[J]. IEEE transactions on industry applications, 1994, 30(5): 1333-1338.

[4] Wenbin Deng, Jae-Won Lee and Hyuk-Jin Lee. Kinematics Simulation and Control of a New 2 DOF Parallel Mechanism Based on Matlab/SimMechanics[J]. In the Proceedings of the ISECS International Colloquium on Computin, Communication, Control, and Management(CCCM 2009), 2009,2:233-236.

[5] H.Khan, S.Abou and N.Sepehri. Fuzzy control of a new type of piezoelectric direct drive electro-hydraulic servo valve[J]. Proceedings of the Fourth International Conference on Machine Learning and Cybernetics, Tokyo, August, 2005: 819-824. 